УДК

\title{
БЫСТРОДЕЙСТВУЮЩИЙ ПАРАЛЛЕЛЬНЫЙ ФИЛЬТР ЧАСТИЦ ДЛЯ СЛЕПОГО РАЗДЕЛЕНИЯ РСМА СИГНАЛОВ
}

\author{
ФЕНГ Х., ГАО Й.
}

Сычуаньский университет,

Китай, Чэнду, 610065, Сычуань

\begin{abstract}
Аннотация. В статье предложен усовершенствованный алгоритм быстродействующего параллельного фильтра частиц (particle filter) для слепого разделения РСМА-сигналов путем использования характеристик параллелизма фильтра частиц с помощью кластерной компьютерной системы, построенной с использованием распределенного вычислительного сервера пакета Matlab и набора инструментальных средств для параллельных вычислений пакета Matlab. Результаты моделирования показывают, что параллельный алгоритм способен быстро и эффективно производить слепое разделение РСМА-сигналов. Кроме того, он значительно сокращает время разделения без снижения эффективности работы алгоритма и улучшает работу системы в режиме реального времени.
\end{abstract}

Ключевые слова: РСМА; совместный доступ к полосе парой несущих; слепое разделение; параллельный фильтр частиц

\section{1. ВВЕДЕНИЕ}

Совместный доступ к полосе парой несущих РCMA (paired carrier multiple access) — это вид системы спутниковой связи, предложенный Марком в 1998 году [1]. Особенность этой системы, позволяющая осуществлять связь на обеих сторонах с той же частотой сигнала в то же время, приводит к удвоению коэффициента использования полосы и повышает способность системы избегать помех. В случае автономной (без приемоответчика) связи или в случае радиотехнической разведки, в результате влияния многих факторов третья сторона часто не имеет априорной информации о каком-либо сигнале. Информация может быть получена только в виде смешанного сигнала, из которого необходимо восстановить дуплексный (двусторонний) символ, содержащийся в этом сигнале, либо передаваемую информацию. Как таковой, это вопрос для системы с одноканаль- ным слепым разделением источников информации [2].

Одноканальное слепое разделение источников имеет много неизвестных переменных, и обычно не может быть решено математически. Однако сигналы связи имеют ограниченные символьные характеристики, и могут быть точно описаны путем использования символов и параметров. Если указанные характерные особенности использовать в полной мере, это позволит улучшить одноканальное выделение РСМА-сигнала.

В последние годы исследователи разработали несколько специальных алгоритмов для одноканального слепого разделения источников смешанных сигналов одинаковой частоты [3-7]. Среди них следует назвать фильтр частиц (particle filter) [8], представляющий собой мощное инструментальное средство для нелинейной и негауссовой оценки состояния. Ука- 


\section{БИБЛИОГРАФИЧЕСКИЙ СПИСОК}

1. Dankberg M. Paired carrier multiple access (PCMA) for satellite communication / Mark Dankberg // Communications Satellite Systems : 17th AIAA Int. Conf. and Exh. ICSSC, Jan. 1998, Honolulu, Hawaii : 
proc. - 1998. - P. 787-791. — DOI : $10.2514 / 6.1998-$ $\underline{1398 .}$.

2. Warner E. S. Single-channel blind signal separation of filtered MPSK signals / E. S. Warner, I. K. Proudler // IEE Proc. Radar, Sonar and Navigation. - Dec. 2003. - Vol. 150, No. 6. - P. 396-402. - DOI : $\underline{10.1049 /}$ ip-rsn:20031007.

3. Heidari $S$. Co-channel interference mitigation in the time-scale domain: the CIMTS algorithm / S. Heidari, C. L. Nikias // IEEE Trans. Signal Process. - Sept. 1996. - Vol. 44, No. 9. - P. 2151-2162. - DOI : $\underline{10.1109 /}$ $\underline{78.536673}$.

4. Liu $K$. Single-channel blind separation of co-frequency MPSK signals / K. Liu, H. Li, X. Dai, X. Xu // Commun., Internet, and Inf. Technology : Int. Conf., Nov. 2006, St. Thomas, USVI, USA : proc. - 2006. — P. $42-46$.

5. Particle filtering based single-channel blind separation of co-frequency MPSK signals / Tu Shilong, Cheng Shaohe, Zheng Hui, Wan Jian // Intelligent Signal Processing and Communication Systems : Int. Symp. ISPACS, 28 Nov.-1 Dec. 2007, Xiamen, China : proc. IEEE, 2007. — P. 582-585. — DOI : 10.1109/ispacs. 2007.4445954.

6. Shilong T. Single-channel blind separation of two QPSK signals using per-survivor processing / Tu Shilong, Zheng Hui, Gu Na // Circuits and Systems : IEEE Asia Pacific Conf. APCCAS, 30 Nov.-3 Dec. 2008, Macao, China : proc. - IEEE, 2008. - P. 473-476. — DOI : 10.1109/apccas.2008.4746063.

7. Guo Y. Single-mixture source separation using dimensionality reduction of ensemble empirical mode decomposition and independent component analysis / Yian Guo, Shuhua Huang, Yongtang Li // Circuits, Syst.
Signal Process. - Dec. 2012. - Vol. 31, No. 6. - P. 2047-2060. — DOI : 10.1007/s00034-012-9414-1.

8. A tutorial on particle filters for online nonlinear/non-Gaussian Bayesian tracking / M. S. Arulampalam, S. Maskell, N. Gordon, T. Clapp // IEEE Trans. Signal Process. - Feb. 2002. - Vol. 50, No. 2. P. 174-188. - DOI : 10.1109/78.978374.

9. Novel algorithm of interference time delay estimation in PCMA system / Shao-He Chen, Jian Wan, Shi-Long Tu, Hui Zheng // J. System Simulation. - Nov. 2008. - Vol. 20, No. 21. - P. 5774-5777. - URL : http://en.cnki.com.cn/Article en/CJFDTOTAL-XTFZ20 0821012.htm.

10. A sequential Monte Carlo method for blind signal separation using the difference of time delays / Shao-He Chen, Shi-Long Tu, Jian Wan, Hui Zheng // TENCON : IEEE Region 10 Conf., 30 Oct.-2 Nov. 2007, Taipei, Taiwan, China : proc. - IEEE, 2007. - P. 1-4. - DOI : 10.1109/TENCON.2007.4428845.

11. Marshall $A$. $W$. The use of multi-stage sampling schemes in Monte Carlo computations / Andy W. Marshall // Monte Carlo Methods : Symp., Jun. 1954, University of Florida : proc. - 1954. - P. 123-140.

12. Doucet A. On sequential Monte Carlo sampling methods for Bayesian filtering / Arnaud Doucet, Simon Godsill, Christophe Andrieu // Statistics Comput. - Jul. 2000. - Vol. 10, No. 3. - P. 197-208. — DOI : 10.1023/ A:1008935410038.

13. Gordon N. J. Novel approach to nonlinear/nonGaussian Bayesian state estimation / N. J. Gordon, D. J. Salmond, A. F. M. Smith // IEE Proc. F (Radar and Signal Process.). - Apr. 1993. - Vol. 140, No. 2. - P. 107-113. — DOI : 10.1049/ip-f-2.1993.0015. 